\title{
An Improved Shape Descriptor Using Bezier Curves
}

\author{
Ferdous Ahmed Sohel ${ }^{\star}$, Gour C. Karmakar, and Laurence S. Dooley \\ Gippsland School of Information Technology, Monash University, \\ Churchill, Victoria - 3842, Australia \\ Ferdous.Sohel@infotech.monash.edu.au
}

\begin{abstract}
Existing shape description techniques using Bezier curves do not adequately consider the domain specific shape information such as the cornerity or gradualness of a shape in the control point generation process. This can lead to large distortion in shape representation even when a large descriptor is used. This paper addresses the issue by introducing a novel improved shape descriptor using Bezier curves (ISDBC) algorithm which divides a shape into segments depending on the cornerity and generates the control points for the segments based on shape information. It also provides an efficient control point encoding strategy which exploits the inherent periodic nature of the distances between consecutive control points. The performance of the ISDBC algorithm has been rigorously tested upon a number of arbitrary shapes, with both quantitative and qualitative results confirming its superiority over existing algorithms.
\end{abstract}

\section{Introduction}

A Bezier curve (BC) is defined by a set of control points (CPs), with the number and orientation of these points governing the shape of the curve. It is costly however, to represent a complex shape using a single BC, since a high degree $\mathrm{BC}$ generation is computationally expensive, with the calculation of the CPs alone incurring substantial overheads. To reduce this computational complexity, composite BC [1] has been used to represent more complex shapes, whereby the whole shape is divided into a number of segments, with each individually represented by a $\mathrm{BC}$.

Cinque et al. 2] divided a shape into a priori number of segments containing equal number of shape points and also distributed the CPs evenly over the shape irrespective of the shape complexity. Since the division into segments and the generation of CPs consider only the number of shape points, not the shape complexity, it can produce large distortion even with a large number of segments. To overcome this problem Sohel et al. proposed a generic shape descriptor using Bezier curves (SDBC) [3], which had introduced the concept of significant points and supplementary points considering the domain specific information on the shape. However, since the segments are of equal length in [2]

\footnotetext{
^ Corresponding author.
} 
and [3], they cannot address cornerity and repetitions (loops) of the points on the shape due to the fact that segment ends are not necessarily at the corner points. Composite BC has been used in [4] and [5] to describe the outline of Arabic and Chinese characters respectively and in [6] to model the active shape of lips. Though in these algorithms the shape is divided into a number of segments considering the cornerity of the shape at shape points, they do not consider the shape information in each segment to compute the control points. For each segment, respective segment end points are considered the start and end CPs and the middle CPs are calculated in different approaches. In [5] and [6], the locations of the middle CPs are calculated, and hence highly depended, on the tangents at the segment endpoints. While [6] gets the control a point at the intersections of the tangents, [5] calculates these using computationally expensive trial-and-error methods. Though 4 has adopt an approach to minimise the distortion, it may require further divisions of the segments to keep the distortion low. Moreover, one common problem of these algorithms is that they allow CPs to be out side of the shape and consequently, the descriptor length can be increased.

As CPs describe the shape of any object, efficient encoding of CPs will reduce the descriptor length and hence reduce the communication cost and improve efficiency and quality. While 2] adopted a parametric means of encoding the $\mathrm{CPs}$, there is no explicit way to encode them in [4, [5] and [6. The parametric descriptor of 2 consists of the absolute coordinate values of the first and the fourth CPs, and the angle of direction and magnitude of distance of the second and the third CPs respectively from the first and the fourth CPs. Both distance and angle were encoded as floating point numbers, which means it is unsuitable for very low bit rate video applications, such as video streaming over the Internet, video-on-demand and mobile video transmission for hand-held devices, where there is an innate bandwidth limitation, so alternative methods to reduce the bit rate must be explored. In [3] for equi-length segments, a dynamic fixed length coding (DFLC) has been introduced which reduces the descriptor length utilising the periodic interval between CPs.

This paper presents an improved shape descriptor using Bezier curves (IS$D B C$ ) which seeks to reduce distortion by defining a new strategy for CP generation and use an efficient $\mathrm{CP}$ coding scheme. This $\mathrm{CP}$ generation strategy improves SDBC [3] by dividing the shape into segments depending on the cornerity at the shape points rather than considering equi-length segments, along with considering the domain specific shape information. The CP coding strategy capitalises the periodic nature of the distances between CPs and uses a modified form of the DFLC approach. Performance of the ISDBC algorithm has been extensively tested upon shapes having sharp corners (e.g., fish, Arabic character) and loops (e.g., lip) and both quantitative and qualitative results confirm its improvement compared with the aforementioned shape description methods.

The rest of this paper is organised as follows: Section 2 presents the new ISDBC shape description framework including CP generation and coding strategies. Experimental results are presented in Section 3, confirming the improved performance of the ISDBC model, with some conclusions drawn in Section 4. 


\section{Improved Shape Description Scheme}

The proposed shape description framework comprises two major parts. Firstly, calculation of CPs for each segment and secondly, a modified DFLC coding strategy for CPs based on a combination of Freeman chain code and run-length coding. These are respectively detailed in the following two subsections.

\subsection{Control Point Determination}

In the first step of $\mathrm{CP}$ generation the shape is divided into segments at the corner points on the shape. Bius-Tiu corner detection [7 method has been used in ISDBC since it produces the closest result to a human viewer. For each segment, CPs are selected from shape points, however, rather than considering all shape points in calculating CPs, concept of significant and supplementary points of [3] is used, so that regions having more rapidly changing shape features are given more emphasis than flat regions. Actually, significant points are the least number of shape points that can generate the original shape without distortion. This takes account of domain specific shape information during $\mathrm{CP}$ generation process and also means that consecutive significant points will not necessarily be separated by 1 pel as with shape points. The larger the distance between consecutive significant points, the greater will be their influence upon the shape. In these situations, shape descriptions based only on significant points can produce higher distortion because influential significant points may be excluded from being CPs. To reduce the likelihood of losing influential significant points as CPs, supplementary points are inserted at equal distances between the significant points. Let the combination of significant and supplementary points be referred to as potential boundary points (PBP) and these will be used in calculation of CPs. Note, the greater the number of supplementary points, PBP tends towards the original shape points, while fewer supplementary points may not be able to represent the influential significant points adequately. To balance these two extrema, average distance between consecutive significant points over the entire shape is used as the metric to insert supplementary points. The whole process can mathematically be explained as follows:

The segments can be represented as $S_{i}=\left\{s_{i, 1}, s_{i, 1}, \cdots, s_{i,\left|S_{i}\right|}\right\}_{1 \leq i \leq N}$ where $N$ is the number of segments and $\left|S_{i}\right|$ is the number of shape points in the $i^{\text {th }}$ segment and $s_{i,\left|S_{i}\right|}=s_{(i+1) \% N, 1}$. Thus, if the significant points of $i^{\text {th }}$ segment is denoted as $\operatorname{Sig}_{i}, \operatorname{Sig}_{i}=\left\{\operatorname{sig}_{i, 0}, \operatorname{sig}_{i, 1}, \cdots, \operatorname{sig}_{i,\left|\operatorname{Sig}_{i}\right|}\right\} \subseteq S_{i, 1 \leq i \leq N}$ where $\left|S i g_{i}\right|$ is the cardinality of set $S i g_{i}$. If $d\left(s i g_{i, k-1}, s i g_{i, k}\right)$ denotes the Euclidean distance between $s_{i, k-1}$ and $s i g_{i, k}$, average distance between significant points over the entire shape is $d_{\text {avg }}=\frac{1}{N} \sum_{i=1}^{N} \frac{1}{\mid \text { Sig }_{i} \mid} \sum_{k=2}^{\left|\operatorname{Sig}_{i}\right|} d\left(s i g_{i, k-1}, s_{i g_{i, k}}\right)$. When $d\left(s_{i, k-1}, s_{i, g_{i, k}}\right)>d_{\text {avg }}$, supplementary points are inserted between $s i_{i, k-1}$ and $s i g_{i, k}$. The first point $\left(s p_{1}\right)$ is placed at a distance of $d_{a v g}$ from $s i g_{i, k-1}$ and if $d\left(s p_{1}, s i g_{i, k}\right)>d_{\text {avg }}$, then a further supplementary point is inserted at distance $d_{\text {avg }}$ from $\left(s p_{1}\right)$. This process continues until the distance between two consecutive significant (one of which may be a supplementary point) points is $\leq d_{\text {avg }}$. PBP for each segment can be defined as $B_{i}=\left\{b_{i, 1}, b_{i, 1}, \cdots, b_{i,\left|B_{i}\right|}\right\}, 1 \leq i \leq N$ and 
is used to calculate CPs. In this paper, cubic BC is used for shape description as lower-order curves are too inflexible to represent the shape, while higher degree curves introduce unwanted oscillations and higher computation overhead. Following from technique [2] CPs of cubic BC for the $i^{\text {th }}$ segment can be calculated as: $v_{i, 0}=b_{i, 1} ; v_{i, 1}=b_{i,\left\lceil\frac{\left|B_{i}\right|}{4}\right\rceil} ; v_{i, 2}=b_{i,\left\lceil\frac{3 *\left|B_{i}\right|}{4}\right\rceil} ; v_{i, 3}=b_{i,\left|B_{i}\right|}$.

\subsection{Control Point Encoding}

DFLC encodes the control point differentially, where the direction of a CP from its previous (immediate) $\mathrm{CP}$ is coded using the chain code, with the distance between them being the run-length of the code. An 8-bit code is used for the directions and for the length it had utilised the periodic nature of intervals between CPs. DFLC considered the segments to be of equal length, however, in ISDBC the segments are of variable length in terms of PBP. So the DFLC is required to be modified. In the following paragraph a suitably modified DFLC has been presented.

Modified dynamic fixed length coding: There are periodic intervals between CPs in terms of PBPs. Consider the $i^{t h}$ segment, if $l_{i}$ is the number of PBP between the first and second CPs, the distance between the second and third CPs is $2 * l_{i}$ points and the distance between the third and fourth CPs is also $l_{i}$ points. The same thing is true for any segment. So if the maximum length of all $l_{i} s$ is allotted for every $l_{i}$, all CPs can be fitted into a frame consisting of only one $l$, instead of several $l_{i} s$. Now $z$ is the largest number of PBP in a segment rather than the equal number of approximated boundary points in SDBC. So $z=\max _{1 \leq i \leq N}\left|B_{i}\right|$ and $l=\left\lceil\frac{z}{4}\right\rceil$. The maximum length of $l, L=l * d_{\text {avg }}$ and $L_{1}=\lceil l g L\rceil$ bits require to encode it. Similarly, to encode the length $2 * L$ requires $L_{1}+1$ bits. Bezier CPs encoding uses the periodic pattern shown in the next table, where Dir is the direction bits of a particular CP. The first segment comprises the absolute location of the first PBP followed by $L_{1}$ bits and its direction after which comes $L_{1}+1$ bits with direction and finally $L_{1}$ bits with direction. Each subsequent segment will not require a starting point and can be defined in ordered sequence of $L_{1}, L_{1}+1, L_{1}$ with directions as shown in the following tabular.

\begin{tabular}{|l|l|l|l|l|l|l|l|l|l|l|}
\hline $\begin{array}{l}\text { 4-bits: } \\
\text { length of } L_{1}\end{array}$ & $\begin{array}{l}\text { Starting } \\
\text { point }\end{array}$ & $\begin{array}{l}\text { Dir } \\
+L_{1}\end{array}$ & $\begin{array}{l}\text { Dir } \\
+L_{1}+1\end{array}$ & $\begin{array}{l}\text { Dir } \\
+L_{1}\end{array}$ & $\begin{array}{l}\text { Dir } \\
+L_{1}\end{array}$ & $\begin{array}{l}\text { Dir } \\
+L_{1}+1\end{array}$ & $\begin{array}{l}\text { Dir } \\
+L_{1}\end{array}$ & $\cdots$ & $\begin{array}{l}\text { Dir } \\
+L_{1}\end{array}$ & $\begin{array}{l}\text { Dir } \\
+L_{1}+1\end{array}$ \\
\hline & \multicolumn{3}{|c|}{ First segment } & Next segment & $\cdots$ & Last segment \\
\hline
\end{tabular}

It is noteworthy that, the leading 4-bits in the encoded sequence are reserved to represent the length of $L_{1}$. Normally 4 bits are sufficient to denote the length of $L_{1}$. Using this, a distance of up to $2^{16}$ pel for $L_{1}$ can be encoded, thus the segment length can be up to $4 * 2^{16}$ pel.

Decoding the shape information: Due to the parametric representation of the encoded information and its periodicity, the decoder knows the length and hence the delimiter of each parameter, so it can correctly parse these parameters and reconstruct the shape using them. 


\section{$3 \quad$ Results and Analysis}

The widely-used shape distortion measurement metrics $D_{\max }$ and $D_{m s}$ of 8 were used respectively for the peak and mean-square distortions for numerical analysis. The performance of ISDBC was tested upon the popular object shapes in the literature. Figure 1 shows comparative presentation of the decoded shape by the descriptors with 5 segments on the lip object having loops. The numerical results, along with for some other shapes, are presented in Table 1. Table 1 shows that both SDBC and algorithm in [2] produced large $D_{\max }$ of 12.1 and 10.2 pel respectively and $D_{m s}$ of 7.5 and $5.4 \mathrm{pel}^{2}$ respectively for the lip shape while ISDBC showed better results of $1.5 \mathrm{pel}$ and $0.9 \mathrm{pel}^{2}$ distortions; this is due to considering the cornerity and loops on the shape in ISDBC. In fact, ISDBC produced the lowest distortion among all the methods (including those also consider curvature information in dividing a shape into segments) and this is because of considering the domain specific shape information in CP generation. The results on the fish shape using the cited 2]-6 techniques with 7 segments are compared in Figure 2 and Table1, For instance, ISDBC produced $D_{\max }$ and $D_{m s}$ of $3.85 \mathrm{pel}$ and $2.76 \mathrm{pel}^{2}$ respectively. From the results, it is evident that ISDBC produced the lowest distortions and thus outperformed the aforementioned shape descriptors using BC. A same conclusion is true for the Arabic character which has been used in [4].

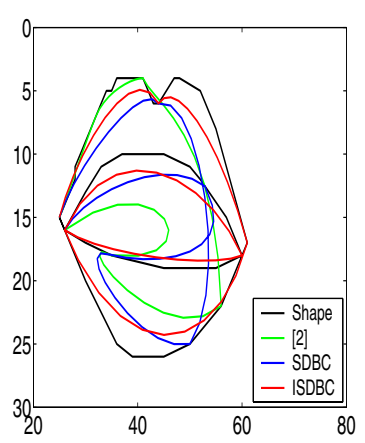

Fig. 1. Results for lipshape used in 6]
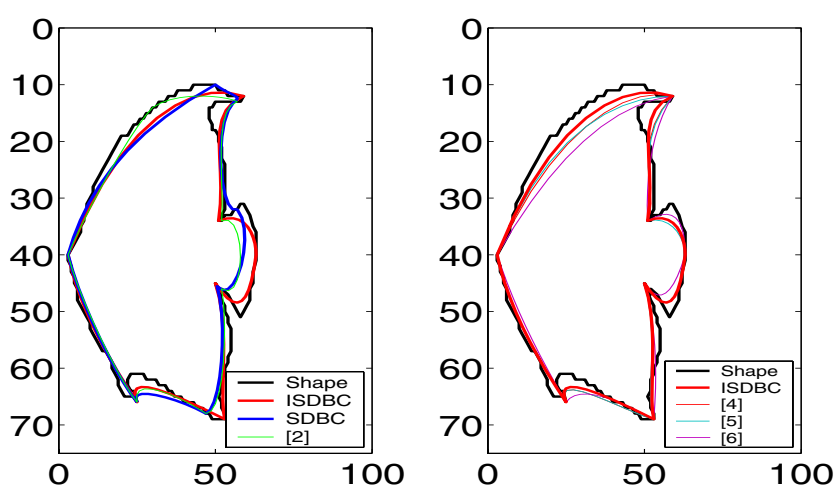

Fig. 2. Comparative results for fish extracted from [2]

For the descriptor length, it was assumed that coordinate values would require one byte each, also despite of the direction and magnitudes in 2 are floating point numbers, only one byte was assumed for each. Thus for fish shape with 7 segments, the descriptor length was 340 bits while ISDBC required only 267 bits, with $z=35$ and $d_{\text {avg }}=1.8$ pel, so $L_{1}=4$ bits. Thus there is a reduction of $20 \%$ in the descriptor size. Moreover, for each additional segments, 2] required 48 bits more, while it is 37 bits for ISDBC and hence, about $23 \%$ improvement for each additional segment. ISDBC is computationally efficient over 
Table 1. Distortion in shape representations (unit: $D_{\max }=p e l, D_{m s}=p e l^{2}$ )

\begin{tabular}{|c|c|c|c|c|c|c|}
\hline Object $\rightarrow$ & \multicolumn{2}{|c|}{ Fish of [2] } & \multicolumn{2}{|c|}{ Arabic of [4] } & \multicolumn{2}{|c|}{ Lip of $[6]$} \\
\hline Technique & $D_{\max }$ & $\mathrm{D}_{\mathrm{ms}}$ & $D_{\max }$ & $\overline{D_{m s}}$ & $D_{\max }$ & $\mathbf{D}_{\mathrm{ms}}$ \\
\hline$\overline{\mathrm{ISDBC}}$ & 3.85 & 2.76 & 1.2 & 0.83 & 1.5 & 0.9 \\
\hline SDBC 3 & 5.4 & 3.6 & 1.9 & 1.25 & 12.1 & 7.5 \\
\hline 2 & 6.0 & 4.3 & 2.1 & 1.35 & 10.2 & 5.4 \\
\hline 4 & 4.22 & 3.34 & 1.3 & 0.95 & 1.65 & 1.05 \\
\hline 5 & 3.9 & 3.46 & 1.35 & 1.2 & 1.7 & 1.2 \\
\hline 6 & 6.05 & 6.55 & 2.0 & 1.4 & 1.8 & 1.5 \\
\hline
\end{tabular}

[4- [6], because of the process of CP generation and coding. However, there is a little increase in computational time requirement over [2]-3] which is secondary when compared with the improvement in the rate-distortion sense.

\section{Conclusions}

While Bezier curves have been applied in many different applications including describing object shapes, a critical aspect in using them is the proper selection of the segments and control points. This paper has presented an improved shape descriptor using Bezier curves (ISDBC) algorithm which provides a novel strategy in defining the control points along with dividing the shape into segments by considering domain specific information concerning the shape and an efficient strategy for encoding the control points. Both qualitative and quantitative results have shown the improved performance of ISDBC compared with existing shape description techniques.

\section{References}

1. R. H. Bartels, J.C.B., Barsky, B.A.: An Introduction to Splines for use in Computer Graphics and Geometric Modeling. Morgan Kaufmann Publishers (1987)

2. L. Cinque, S.L., Malizia, A.: Shape description using cubic polynomial bezier curves. Pattern Recognition Letters, Elsevier Science Inc. (1998) 821-828

3. F.A. Sohel, G.K., Dooley, L.: A generic shape descriptor using bezier curves. In: Int. Conf. on Info. Tech.: New Trends in Image Proc., ITCC. Volume II. (2005) 95-100

4. Sarfraz, M., Khan, M.A.: Automatic outline capture of arabic fonts. Information Sciences, Elsevier Science Inc. (2002) 269-281

5. H. M. Yang, J.J.L., Lee, H.J.: A bezier curve-based approach to shape description for chinese calligraphy characters. In: Int. Conf. Doc. Anal. Recog. (2001) 276-280

6. I. Shdaifat, R.G., Langmann, D.: Active shape lip modeling. In: Proc. of International Conference on Image Processing, ICIP-2003. (2003) 875-878

7. Beus, H.L., Tiu, S.S.H.: An improved corner detection algorithm based on chain coded plane curves. Pattern Recognition 20 (1987) 291-296

8. Schuster, G.M., Katsaggelos, A.K.: Rate-Distortion Based Video CompressionOptimal Video Frame Compression and Object Boundary Encoding. Kluwer Academic Publishers (1997) 